\title{
Knowledge and Practice of Anemia among pregnant women attending antenatal clinic in Dr. Prabhakar Kore hospital, Karnataka-A Cross sectional study.
}

\author{
Rajeev Kumar Yadav ${ }^{1}$, M.K Swamy ${ }^{2}$, Bijendra Banjade ${ }^{3}$ \\ ${ }^{I}$ P.G student, M.P.H, Department of public Health, J.N. Medical College, KLE University, India. \\ ${ }^{2}$ Professor, MD, Department of Genecology and Obstetrics, J. N. Medical College, KLE University,India.. \\ ${ }^{3}$ BPH, (MPH), Department of public Health, J.N. Medical College, KLE University, India.
}

\begin{abstract}
Background and Objectives: Anemia in pregnancy is defined by World Health Organization (WHO) as a haemoglobin concentration below $11 \mathrm{~g} / \mathrm{dl}$. Iron-deficiency anemia is the most common form of malnutrition in the world and is the eighth leading cause of disease in girls and pregnant women in developing countries. Materials and Methods: A cross sectional study was conducted Obstetrics and Gynaecological outpatient department of K.L.E.S hospital by taking a total of 400 pregnant women. Results: Out of 400 respondent's majority, $60.5 \%$ of the pregnant women were aged between 20-24 years.. Majority 62.7\% of the respondents had registered their pregnancy in $1^{\text {st }}$ trimester. Majority $48.5 \%$ of the respondents were in the first gravid. Most of the respondents didn't have any children. The study found that there was significant association between women's education and knowledge regarding cause of anemia, knowledge regarding sign and symptoms regarding anemia, knowledge regarding proper diet to prevent anemia, knowledge regarding prevention and treatment of anemia, preventive practice regarding anemia at $p<0.001$.Conclusion: The study result showed that knowledge regarding cause of anemia, sign and symptoms of anemia, proper diet to prevent anemia was poor. Knowledge regarding prevention and treatment of anemia, knowledge regarding preventive practice of anemia was good.
\end{abstract}

Key Words: Anemia, Pregnant women, ANC, Knowledge, Practice

\section{Introduction:}

Anemia in pregnancy is defined by World Health Organization (WHO) as a hemoglobin concentration below $11 \mathrm{~g} / \mathrm{dl}{ }^{1}{ }^{1}$ Iron-deficiency anemia is the most common form of malnutrition in the world and is the eighth leading cause of disease in girls and pregnant women in developing countries. Women's health is central to the survival of the society as they give beginning to the new life on the earth and cares for all the family members. ${ }^{2}$ Both developed and developing countries are affected by anemia. It has been global public health problem with major consequences for human health. It affects people of all age groups but its prevalence is more in pregnant women and young children. According to WHO, anemia is classified as mild degree (Hb 9.0$11.0 \mathrm{~g} \backslash \mathrm{dl})$, moderate $(7.0-9.0 \mathrm{~g} \backslash \mathrm{dl})$ and severe ( $4.0-7.0 \mathrm{~g} \backslash \mathrm{dl}){ }^{3}$

In developing countries the major cause of anemia is hook worm infestation because many developing countries are located in tropical climate. In this region, low income country like India faces the problem of nonavailability of iron rich food. ${ }^{4}$ WHO estimates that the prevalence of anemia ranges from $40-60 \%$ in the developing countries. Half of those who are suffering from anemia are supposed to be suffering from iron deficiency anemia (IDA). The WHO has estimated that the prevalence of anemia in developed and developing countries in pregnant women is $14 \%$ in developed and $51 \%$ in developing countries. For example in India, anemia was estimated at $65-75 \%{ }^{2}$ Pregnancy anemia is one of the important public health problems not only in India but also in most of the South East Asian countries. ${ }^{5}$

The prevalence of anemia is very high i.e (33-75\%) in developing countries to that of $15 \%$ in developed countries. According to National Family Health survey-III (2005-2006) prevalence of anemia among pregnant women in India is 58\% which is higher as compare to the previous survey (NFHS - II). In India most of the population is predominantly vegetarian and food stuff of Indian diet contain significant amount of phosphates, oxalates. ${ }^{6}$

Very few researches are done in India regarding knowledge and practice of anemia in pregnant women. This research will be fruitful to formulate the policy regarding the vulnerable group of society. Hence, this study was done to assess the knowledge and practice among the pregnant women who are attending antenatal clinic in Dr. Prabhakar Kore Hospital. 


\section{Material And Methods:}

A cross-sectional study was conducted in the "Obstetrics and Gynecological out Patient Department of K.L.E.S Hospital" for a period of 1 year (Feb.2013-Jan.2014) among registered pregnant women attending antenatal clinics. Sample size was calculated by the formula $\left(n=4 \mathbf{p q} / \mathbf{d}^{2}\right)(\mathrm{p}=$ Prevalence i.e. $50 \%, q=100-p$, $d=10 \%$ error of p i.e. $10 / 100 * 50=5$ ). The total sample was 400 .

A total of 400 pregnant women attending ANC clinic were randomly selected and included in the Study. All the registered pregnant mothers, those are attending the antenatal clinics were included in the study. Pregnant mother admitted in antenatal ward and associated with complication i.e. bad obstetric history, High risk pregnancies were excluded from the study.

A structured interview schedule was used to collect the required information. Ethical clearance was obtained from Institutional Ethics Committee (IEC) of JNMC, KLEU. A formal permission to conduct the study was obtained from the authorities of the hospital and consent was taken from study participants. The data was entered in SPSS (version 20) and analyzed by using descriptive (percentage, rate) and inferential statistical (chisquare).

\section{Results:}

Majority of the women were in the age group 20-24 years i.e.60.50\%, 26.50\% were in the age group 25-18 years, $8.50 \%$ and $4.50 \%$ women are in the age group 15-19 and 30-34 years respectively. Out of 400 respondents, $37.0 \%$ studied up to secondary level, $30.3 \%$ studied up to primary level, $13.8 \%, 8.3 \%$ studied up to higher secondary and graduate level respectively. 43(10.8\%) women were illiterate. Majority were Hindu i.e. $74.30 \%$ and $25.30 \%$ were Muslim. $67.7 \%$ were living in joint family and $18.3 \%$ in nuclear family. Only $12(3.0 \%)$ were living in extended family. Majority i.e. $86.50 \%$ were housewife, $4.0 \%$ were labor. $62.70 \%$ were registered their pregnancy in first trimester, $31.30 \%$ had registered in second trimester and $6.0 \%$ had registered their pregnancy in third trimester (Table no.1).

$34.80 \%$ had family's income Rs.5000-10000 per month, 30.50\% had family's income less then Rs 5000 per month. $20.80 \%, 9.20 \%, 3.80 \%$ had family income per month Rs.10000-15000, Rs 15000-20000, Rs20000-25000 respectively. Only $1.0 \%$ had family's income more than Rs 25000 per month.

Out of 400 respondents, majority $48.50 \%$ were in first gravid, $33.20 \%$ were in second gravid. $16.0 \%$ and $2.30 \%$ were in third and fourth gravid respectively. It was found that $46.5 \%$ didn't have any child and 39.30 had one child. Similarly, $7.20 \%, 6.70 \%, 0.30 \%$ had two, three and four children respectively.

The study found that there was significant association regarding knowledge about cause of anemia, sign and symptoms, proper diet to prevent, prevention and treatment and preventive practices with women's education (Table no.2,3,4,5,6).

Table no.1: Distribution of Socio-demographic Variables of Respondents $(n=400)$

\begin{tabular}{|c|c|c|c|}
\hline & Variables & \multirow{2}{*}{$\begin{array}{l}\text { No. } \\
34\end{array}$} & \multirow{2}{*}{$\begin{array}{c}\text { Percentage (\%) } \\
8.5 \\
\end{array}$} \\
\hline \multirow{4}{*}{ Age in years } & $15-19$ & & \\
\hline & $20-24$ & 242 & 60.5 \\
\hline & $25-18$ & 106 & 26.5 \\
\hline & $30-34$ & 18 & 4.5 \\
\hline \multirow{5}{*}{ Literacy Status } & Illiterate & 43 & 10.8 \\
\hline & Primary & 121 & 30.3 \\
\hline & Secondary & 148 & 37.0 \\
\hline & Higher secondary & 55 & 13.8 \\
\hline & Graduate & 33 & 8.3 \\
\hline \multirow{3}{*}{ Religion } & Hindu & 187 & 74.3 \\
\hline & Muslim & 102 & 25.3 \\
\hline & Buddhist & 1 & 0.3 \\
\hline \multirow{3}{*}{ Types of Family } & Nuclear & 117 & 18.3 \\
\hline & Joint & 161 & 67.8 \\
\hline & Extended & 12 & 3.0 \\
\hline \multirow{3}{*}{ Occupation } & House wife & 346 & 86.5 \\
\hline & Labor & 16 & 4 \\
\hline & Others & 38 & 9.5 \\
\hline \multirow[b]{2}{*}{ Diet } & Vegetarian & 128 & 32.0 \\
\hline & Non-vegetarian & 162 & 68.0 \\
\hline \multirow{3}{*}{ Registered during } & $1^{\text {st }}$ trimester & 251 & 62.7 \\
\hline & $2^{\text {nd }}$ trimester & 125 & 31.3 \\
\hline & $3^{\text {rd }}$ trimester & 24 & 6.0 \\
\hline
\end{tabular}


Knowledge and practice of anaemia among pregnant women attending antenatal clinic

Table no.2: Association between knowledge regarding cause of anemia and women's education

\begin{tabular}{|c|c|c|c|c|c|c|c|}
\hline \multirow{8}{*}{$\begin{array}{l}\text { Pregnancy creates } \\
\text { large demand of iron } \\
\text { which is needed to } \\
\text { develop the placenta }\end{array}$} & \multirow[t]{2}{*}{ Women's Education } & \multicolumn{2}{|c|}{ Correct } & \multicolumn{2}{|c|}{ Incorrect } & \multicolumn{2}{|c|}{ Total } \\
\hline & & No. & $\%$ & No. & $\%$ & No. & $\%$ \\
\hline & Illiterate & 0 & 0 & 43 & 100 & 43 & 10.75 \\
\hline & Primary & 14 & 11.57 & 107 & 88.42 & 121 & 30.25 \\
\hline & Secondary & 18 & 12.16 & 130 & 87.84 & 148 & 37 \\
\hline & Higher secondary & 1 & 1.81 & 54 & 98.19 & 55 & 13.75 \\
\hline & Graduate & 13 & 39.39 & 20 & 60.61 & 33 & 8.25 \\
\hline & \multicolumn{5}{|c|}{$\chi 2=35.946, \mathrm{Df}=4, \mathrm{p}<0.001$} & 400 & 100 \\
\hline \multirow{6}{*}{$\begin{array}{l}\text { Increase of } \mathrm{HB} \% \text { in } \\
\text { the blood is known as } \\
\text { anemia }\end{array}$} & Illiterate & 0 & 0 & 43 & 100 & 43 & 10.75 \\
\hline & Primary & 5 & 4.3 & 116 & 95.87 & 121 & 30.25 \\
\hline & Secondary & 13 & 8.78 & 135 & 91.22 & 148 & 37 \\
\hline & Higher secondary & 0 & 0 & 55 & 100 & 55 & 13.75 \\
\hline & Graduate & 9 & 27.27 & 24 & 72.73 & 33 & 8.25 \\
\hline & \multicolumn{5}{|c|}{$\chi 2=31.465, \mathrm{DF}=4, \mathrm{p}=<0.001$} & 400 & 100 \\
\hline \multirow{6}{*}{$\begin{array}{l}\text { Anemia in pregnancy is } \\
\text { nutritional disorder }\end{array}$} & Illiterate & 0 & 0 & 43 & 100 & 43 & 10.75 \\
\hline & Primary & 0 & 0 & 121 & 100 & 121 & 30.25 \\
\hline & Secondary & 14 & 9.45 & 134 & 90.55 & 148 & 37 \\
\hline & Higher secondary & 1 & 1.81 & 54 & 98.19 & 55 & 13.75 \\
\hline & Graduate & 8 & 69.69 & 25 & 30.31 & 33 & 8.25 \\
\hline & \multicolumn{5}{|c|}{$\chi 2=39.349, D F=8, p<0.001$} & 400 & 100 \\
\hline \multirow{6}{*}{$\begin{array}{l}\text { Iron is an important } \\
\text { elements required for } \\
\text { Hb for pregnancy }\end{array}$} & Illiterate & 0 & 0 & 43 & 100 & 43 & 10.75 \\
\hline & Primary & 0 & 0 & 121 & 100 & 121 & 30.25 \\
\hline & Secondary & 21 & 14.18 & 127 & 85.82 & 148 & 37 \\
\hline & Higher secondary & 1 & 1.81 & 54 & 98.19 & 55 & 13.75 \\
\hline & Graduate & 17 & 24.24 & 16 & 75.76 & 33 & 8.25 \\
\hline & \multicolumn{5}{|c|}{$\chi^{2}=90.381, D F=4, p<0.001$} & 400 & 100 \\
\hline \multirow{6}{*}{$\begin{array}{l}\text { Major cause of anemia } \\
\text { is malaria }\end{array}$} & Illiterate & 0 & 0 & 43 & 100 & 43 & 10.75 \\
\hline & Primary & 0 & 0 & 121 & 100 & 121 & 30.25 \\
\hline & Secondary & 10 & 6.75 & 138 & 85.82 & 148 & 37 \\
\hline & Higher secondary & 4 & 7.27 & 51 & 98.19 & 55 & 13.75 \\
\hline & Graduate & 9 & 27.27 & 24 & 75.76 & 33 & 8.25 \\
\hline & \multicolumn{5}{|c|}{$\chi^{2}=38.725$, DF $=4, p<0.001$} & 400 & \\
\hline \multirow{6}{*}{$\begin{array}{l}\text { Repeated pregnancy at } \\
\text { a short interval i.e. }<2 \\
\text { yrs cause anemia }\end{array}$} & Illiterate & 0 & 0 & 43 & 100 & 43 & 10.75 \\
\hline & Primary & 2 & 1.65 & 121 & 98.35 & 121 & 30.25 \\
\hline & Secondary & 20 & 13.51 & 128 & 86.49 & 148 & 37 \\
\hline & Higher secondary & 4 & 7.27 & 51 & 92.73 & 55 & 13.75 \\
\hline & Graduate & 13 & 39.39 & 20 & 30.61 & 33 & 8.25 \\
\hline & \multicolumn{5}{|c|}{$\chi^{2}=49.383, \mathrm{DF}=4, \mathrm{p}<0.001$} & 400 & 100 \\
\hline
\end{tabular}

Table no.3: Association between knowledge regarding sign and symptoms of anemia and women's education.

\begin{tabular}{|c|c|c|c|c|c|c|c|}
\hline \multirow{8}{*}{$\begin{array}{l}\text { Tiredness and } \\
\text { weakness are symptoms } \\
\text { of anemia }\end{array}$} & \multirow[t]{2}{*}{ Women's Education } & \multicolumn{2}{|c|}{ Correct } & \multicolumn{2}{|c|}{ Incorrect } & \multicolumn{2}{|c|}{ Total } \\
\hline & & No. & $\%$ & No. & $\%$ & No. & $\%$ \\
\hline & Illiterate & 0 & 0 & 43 & 100 & 43 & 10.75 \\
\hline & Primary & 2 & 1.65 & 119 & 98.35 & 121 & 30.25 \\
\hline & Secondary & 8 & 5.40 & 140 & 94.60 & 148 & 37 \\
\hline & Higher secondary & 14 & 25.45 & 41 & 74.54 & 55 & 13.75 \\
\hline & Graduate & 13 & 39.39 & 20 & 60.61 & 33 & 8.25 \\
\hline & \multicolumn{5}{|c|}{$\chi^{2}=68.23$ DF $=4, p<0.001$} & 400 & 100 \\
\hline \multirow{6}{*}{$\begin{array}{l}\text { Pallor of face are sign } \\
\text { of anemia }\end{array}$} & Illiterate & 0 & 0 & 43 & 100 & 43 & 10.75 \\
\hline & Primary & 17 & 14.04 & 110 & 85.96 & 121 & 30.25 \\
\hline & Secondary & 27 & 18.24 & 121 & 81.76 & 148 & 37 \\
\hline & Higher secondary & 12 & 21.81 & 43 & 78.19 & 55 & 13.75 \\
\hline & Graduate & 12 & 36.36 & 21 & 63.36 & 33 & 8.25 \\
\hline & \multicolumn{5}{|c|}{$\chi^{2}=25.17, \mathrm{DF}=4 \mathrm{p}<0.001$} & 400 & 100 \\
\hline \multirow{6}{*}{$\begin{array}{l}\text { Pallor of eyes is sign of } \\
\text { anemia }\end{array}$} & Illiterate & 0 & 0 & 43 & 100 & 43 & 10.75 \\
\hline & Primary & 13 & 10.74 & 108 & 89.26 & 121 & 30.25 \\
\hline & Secondary & 22 & 14.86 & 126 & 85.10 & 148 & 37 \\
\hline & Higher secondary & 16 & 40.0 & 39 & 60.0 & 55 & 13.75 \\
\hline & Graduate & 8 & 48.48 & 25 & 51.52 & 33 & 8.25 \\
\hline & \multicolumn{5}{|c|}{$\chi^{2}=20.34, \mathrm{DF}=4, \mathrm{p}<0.001$} & 400 & 100 \\
\hline \multirow{7}{*}{$\begin{array}{l}\text { Pallor of tongue is sign } \\
\text { of anemia }\end{array}$} & Illiterate & 0 & 0 & 43 & 100 & 43 & 10.75 \\
\hline & Primary & 0 & 0 & 121 & 100 & 121 & 30.25 \\
\hline & Secondary & 16 & 10.81 & 132 & 91.9 & 148 & 37 \\
\hline & Higher secondary & 16 & 29.09 & 39 & 67.28 & 55 & 13.75 \\
\hline & Graduate & 12 & 36.36 & 21 & 63.64 & 33 & 8.25 \\
\hline & \multicolumn{5}{|c|}{$\chi^{2}=60.35, D F=4, p<0.001$} & 400 & 100 \\
\hline & Illiterate & 0 & 0 & 43 & 100 & 43 & 10.75 \\
\hline
\end{tabular}


Knowledge and practice of anaemia among pregnant women attending antenatal clinic

\begin{tabular}{|c|c|c|c|c|c|c|c|}
\hline \multirow{5}{*}{$\begin{array}{l}\text { Pallor of nails is sign of } \\
\text { anemia }\end{array}$} & Primary & 0 & 0 & 121 & 100 & 121 & 30.25 \\
\hline & Secondary & 12 & 8.10 & 126 & 91.9 & 148 & 37 \\
\hline & Higher secondary & 18 & 32.72 & 47 & 67.28 & 55 & 13.75 \\
\hline & Graduate & 12 & 36.36 & 21 & 63.64 & 33 & 8.25 \\
\hline & \multicolumn{5}{|c|}{$\chi^{2}=46.68, D F=4, p<0.001$} & 400 & 100 \\
\hline \multirow{6}{*}{$\begin{array}{l}\text { Palpitation and } \\
\text { breathing difficulty are } \\
\text { sign of anemia }\end{array}$} & Illiterate & 0 & 0 & 43 & 100 & 43 & 10.75 \\
\hline & Primary & 0 & 0 & 121 & 100 & 121 & 30.25 \\
\hline & Secondary & 18 & 12.16 & 130 & 87.84 & 148 & 37 \\
\hline & Higher secondary & 4 & 7.27 & 51 & 92.73 & 55 & 13.75 \\
\hline & Graduate & 12 & 36.36 & 21 & 63.64 & 33 & 8.25 \\
\hline & \multicolumn{5}{|c|}{$\chi^{2}=44.42, \mathrm{DF}=4, \mathrm{p}<0.001$} & 400 & 100 \\
\hline
\end{tabular}

Table no.4: Association regarding knowledge regarding proper diet to prevent anemia and women's

\begin{tabular}{|c|c|c|c|c|c|c|c|}
\hline \multirow{8}{*}{$\begin{array}{l}\text { A well balance diet } \\
\text { during pregnancy } \\
\text { prevents anemia }\end{array}$} & \multirow[t]{2}{*}{ Women's Education } & \multicolumn{2}{|c|}{ Correct } & \multicolumn{2}{|c|}{ Incorrect } & \multicolumn{2}{|c|}{ Total } \\
\hline & & No. & $\%$ & No. & $\%$ & No. & $\%$ \\
\hline & Illiterate & 10 & 23.3 & 33 & 76.7 & 43 & 10.75 \\
\hline & Primary & 51 & 42.1 & 70 & 57.9 & 121 & 30.25 \\
\hline & Secondary & 87 & 58.8 & 61 & 41.2 & 148 & 37 \\
\hline & Higher secondary & 51 & 92.7 & 4 & 7.3 & 55 & 13.75 \\
\hline & Graduate & 29 & 87.9 & 4 & 12.1 & 33 & 8.25 \\
\hline & \multicolumn{5}{|c|}{$\chi^{2}=72.53, \mathrm{DF}=4, \mathrm{p}<0.001$} & 400 & 100 \\
\hline \multirow{6}{*}{$\begin{array}{l}\text { Green leafy vegetables } \\
\text { and sprouted grains are } \\
\text { rich in iron }\end{array}$} & Illiterate & 0 & 0 & 43 & 100 & 43 & 10.75 \\
\hline & Primary & 54 & 44.6 & 67 & 55.4 & 121 & 30.25 \\
\hline & Secondary & 87 & 58.8 & 61 & 41.2 & 148 & 37 \\
\hline & Higher secondary & 51 & 92.7 & 4 & 7.3 & 55 & 13.75 \\
\hline & Graduate & 25 & 75.8 & 8 & 24.2 & 33 & 8.25 \\
\hline & \multicolumn{5}{|c|}{$\chi^{2}=95.68, \mathrm{DF}=4, \mathrm{p}<0.001$} & 400 & 100 \\
\hline \multirow{6}{*}{$\begin{array}{l}\text { Ragi and Jaggery should } \\
\text { be avoided during } \\
\text { pregnancy }\end{array}$} & Illiterate & 20 & 46.5 & 23 & 53.5 & 43 & 10.75 \\
\hline & Primary & 46 & 38.0 & 75 & 62.0 & 121 & 30.25 \\
\hline & Secondary & 53 & 35.8 & 95 & 64.2 & 148 & 37 \\
\hline & Higher secondary & 32 & 58.2 & 23 & 41.8 & 55 & 13.75 \\
\hline & Graduate & 8 & 24.2 & 25 & 75.8 & 33 & 8.25 \\
\hline & \multicolumn{5}{|c|}{$\chi^{2}=13.07, \mathrm{DF}=4, \mathrm{p}<0.001$} & 400 & 100 \\
\hline \multirow{6}{*}{$\begin{array}{l}\text { Meat is rich source of } \\
\text { iron }\end{array}$} & Illiterate & 25 & 58.1 & 18 & 41.9 & 43 & 10.75 \\
\hline & Primary & 58 & 47.9 & 63 & 52.1 & 121 & 30.25 \\
\hline & Secondary & 73 & 49.3 & 75 & 50.7 & 148 & 37 \\
\hline & Higher secondary & 35 & 63.6 & 20 & 36.4 & 55 & 13.75 \\
\hline & Graduate & 5 & 15.2 & 28 & 84.8 & 33 & 8.25 \\
\hline & \multicolumn{5}{|c|}{$\chi^{2}=21.34, \mathrm{DF}=4, \mathrm{p}<0.001$} & 400 & 100 \\
\hline \multirow{6}{*}{$\begin{array}{l}\text { Liver is rich source of } \\
\text { iron }\end{array}$} & Illiterate & 25 & 58.1 & 18 & 41.9 & 43 & 10.75 \\
\hline & Primary & 50 & 41.3 & 71 & 58.7 & 121 & 30.25 \\
\hline & Secondary & 71 & 48.0 & 77 & 52.0 & 148 & 37 \\
\hline & Higher secondary & 43 & 78.2 & 12 & 21.8 & 55 & 13.75 \\
\hline & Graduate & 5 & 15.2 & 28 & 84.8 & 33 & 8.25 \\
\hline & \multicolumn{5}{|c|}{$\chi^{2}=38.20, \mathrm{DF}=4, \mathrm{p}<0.001$} & 400 & 100 \\
\hline \multirow{6}{*}{$\begin{array}{l}\text { Citrus fruits promotes } \\
\text { absorption of iron }\end{array}$} & Illiterate & 14 & 32.6 & 29 & 67.4 & 43 & 10.75 \\
\hline & Primary & 29 & 24.0 & 92 & 76.0 & 121 & 30.25 \\
\hline & Secondary & 89 & 60.1 & 59 & 39.9 & 148 & 37 \\
\hline & Higher secondary & 39 & 70.9 & 16 & 29.1 & 55 & 13.75 \\
\hline & Graduate & 21 & 63.6 & 12 & 36.4 & 33 & 8.25 \\
\hline & \multicolumn{5}{|c|}{$\chi^{2}=55.63, \mathrm{DF}=4, \mathrm{p}<0.001$} & 400 & 100 \\
\hline
\end{tabular}

Table no.5: Association regarding knowledge about prevention and treatment of anemia and women's

\begin{tabular}{|c|c|c|c|c|c|c|c|}
\hline \multirow{6}{*}{$\begin{array}{l}\text { Regular medical checkup } \\
\text { is necessary during } \\
\text { pregnancy }\end{array}$} & \multirow[t]{2}{*}{ Women's Education } & \multicolumn{2}{|c|}{ Correct } & \multicolumn{2}{|c|}{ Incorrect } & \multicolumn{2}{|c|}{ Total } \\
\hline & & No. & $\%$ & No. & $\%$ & No. & $\%$ \\
\hline & Illiterate & 24 & 55.8 & 19 & 44.2 & 43 & 10.8 \\
\hline & Secondary & 123 & 83.1 & 25 & 16.9 & 148 & 37.0 \\
\hline & Higher secondary & 50 & 90.9 & 5 & 9.1 & 55 & 13.8 \\
\hline & Graduate & 21 & 63.6 & 12 & 36.4 & 33 & 8.2 \\
\hline \multirow{6}{*}{$\begin{array}{l}\text { Daily intake of iron and } \\
\text { folic acid tablet is } \\
\text { necessary }\end{array}$} & Illiterate & 14 & 32.6 & 29 & 67.4 & 43 & 10.8 \\
\hline & Primary & 76 & 62.8 & 45 & 37.2 & 121 & 30.2 \\
\hline & Secondary & 117 & 79.0 & 31 & 21.0 & 148 & 37.0 \\
\hline & Higher secondary & 51 & 92.7 & 4 & 7.3 & 55 & 13.8 \\
\hline & Graduate & 16 & 48.4 & 17 & 51.6 & 33 & 8.2 \\
\hline & \multicolumn{5}{|c|}{$\chi^{2}=56.28, \mathrm{DF}=4, \mathrm{p}<0.001$} & 400 & 100 \\
\hline
\end{tabular}


Knowledge and practice of anaemia among pregnant women attending antenatal clinic

\begin{tabular}{|c|c|c|c|c|c|c|c|}
\hline \multirow{6}{*}{$\begin{array}{l}\text { Adequate treatment is } \\
\text { necessary to treat hook } \\
\text { worm infestation }\end{array}$} & Illiterate & 20 & 46.5 & 23 & 53.5 & 43 & 10.8 \\
\hline & Primary & 77 & 63.6 & 44 & 36.4 & 121 & 30.2 \\
\hline & Secondary & 106 & 71.6 & 42 & 28.4 & 148 & 37.0 \\
\hline & Higher secondary & 39 & 70.9 & 16 & 29.1 & 55 & 13.8 \\
\hline & Graduate & 16 & 48.4 & 17 & 51.6 & 33 & 8.2 \\
\hline & \multicolumn{5}{|c|}{$\chi^{2}=14.07, \mathrm{DF}=4, \mathrm{p}<0.001$} & 400 & 100 \\
\hline \multirow{6}{*}{$\begin{array}{l}\text { Do you know Free iron } \\
\text { tablet is given at time of } \\
\text { pregnancy? }\end{array}$} & Illiterate & 39 & 90.6 & 4 & 9.4 & 43 & 10.8 \\
\hline & Primary & 112 & 92.5 & 9 & 7.5 & 121 & 30.2 \\
\hline & Secondary & 132 & 89.1 & 16 & 10.9 & 148 & 37.0 \\
\hline & Higher secondary & 51 & 92.7 & 4 & 7.3 & 55 & 13.8 \\
\hline & Graduate & 24 & 72.7 & 9 & 27.3 & 33 & 8.2 \\
\hline & \multicolumn{5}{|c|}{$\chi^{2}=11.77, \mathrm{DF}=4, \mathrm{p}<0.001$} & 400 & 100 \\
\hline
\end{tabular}

Table no.6: Association between preventive practice regarding anemia and women's education.

\begin{tabular}{|c|c|c|c|c|c|c|c|}
\hline \multirow{8}{*}{$\begin{array}{l}\text { Have you changed your } \\
\text { normal dietary pattern } \\
\text { during pregnancy? }\end{array}$} & \multirow[t]{2}{*}{ Women's Education } & \multicolumn{2}{|c|}{ Healthy Practice } & \multicolumn{2}{|c|}{ Unhealthy Practice } & \multicolumn{2}{|c|}{ Total } \\
\hline & & No. & $\%$ & No. & $\%$ & No. & $\%$ \\
\hline & Illiterate & 14 & 32.5 & 29 & 67.5 & 43 & 10.75 \\
\hline & Primary & 68 & 56.1 & 53 & 43.9 & 121 & 30.25 \\
\hline & Secondary & 102 & 68.9 & 46 & 31.1 & 148 & 37 \\
\hline & Higher secondary & 42 & 76.3 & 13 & 23.7 & 55 & 13.75 \\
\hline & Graduate & 28 & 84.8 & 5 & 15.2 & 33 & 8.25 \\
\hline & \multicolumn{5}{|c|}{$\chi^{2}=32.83, \mathrm{DF}=4, \mathrm{p}<0.001$} & 400 & 100 \\
\hline \multirow{6}{*}{$\begin{array}{l}\text { Do you include green } \\
\text { leafy vegetable in your } \\
\text { diet every day? }\end{array}$} & Illiterate & 43 & 100 & 0 & 0 & 43 & 10.75 \\
\hline & Primary & 113 & 93.3 & 8 & 6.7 & 121 & 30.25 \\
\hline & Secondary & 141 & 95.2 & 7 & 4.8 & 148 & 37 \\
\hline & Higher secondary & 55 & 100 & 0 & 0 & 55 & 13.75 \\
\hline & Graduate & 25 & 75.7 & 8 & 24.3 & 33 & 8.25 \\
\hline & \multicolumn{5}{|c|}{$\chi^{2}=27.25, \mathrm{DF}=4, \mathrm{p}<0.001$} & 400 & 100 \\
\hline \multirow{6}{*}{$\begin{array}{l}\text { Do you eat sprouted } \\
\text { grains in your diet every } \\
\text { day? }\end{array}$} & Illiterate & 43 & 100 & 0 & 0 & 43 & 10.75 \\
\hline & Primary & 112 & 92.5 & 9 & 7.5 & 121 & 30.25 \\
\hline & Secondary & 132 & 89.1 & 16 & 10.9 & 148 & 37 \\
\hline & Higher secondary & 55 & 100 & 0 & 0 & 55 & 13.75 \\
\hline & Graduate & 25 & 75.7 & 8 & 24.3 & 33 & 8.25 \\
\hline & \multicolumn{5}{|c|}{$\chi^{2}=21.35, D F=4, p<0.001$} & 400 & 100 \\
\hline \multirow{6}{*}{$\begin{array}{l}\text { Do you include fiber } \\
\text { rich food frequently? }\end{array}$} & Illiterate & 34 & 79.0 & 9 & 21.0 & 43 & 10.75 \\
\hline & Primary & 112 & 92.5 & 9 & 7.5 & 121 & 30.25 \\
\hline & Secondary & 138 & 93.2 & 10 & 6.8 & 148 & 37 \\
\hline & Higher secondary & 55 & 100 & 0 & 100 & 55 & 13.75 \\
\hline & Graduate & 28 & 84.8 & 5 & 15.2 & 33 & 8.25 \\
\hline & \multicolumn{5}{|c|}{$\chi^{2}=16.69, \mathrm{DF}=4, \mathrm{p}<0.001$} & 400 & 100 \\
\hline \multirow{6}{*}{$\begin{array}{l}\text { Do you use ragi in your } \\
\text { diet? }\end{array}$} & Illiterate & 10 & 23.2 & 33 & 76.8 & 43 & 10.75 \\
\hline & Primary & 94 & 77.6 & 27 & 22.4 & 121 & 30.25 \\
\hline & Secondary & 98 & 66.2 & 50 & 33.8 & 148 & 37 \\
\hline & Higher secondary & 30 & 54.5 & 25 & 45.5 & 55 & 13.75 \\
\hline & Graduate & 24 & 72.7 & 9 & 27.3 & 33 & 8.25 \\
\hline & \multicolumn{5}{|c|}{$\chi^{2}=44.36, \mathrm{DF}=4, \mathrm{p}<0.001$} & 400 & 100 \\
\hline \multirow{6}{*}{$\begin{array}{l}\text { Do you use jiggery in } \\
\text { your diet? }\end{array}$} & Illiterate & 14 & 32.5 & 29 & 67.5 & 43 & 10.75 \\
\hline & Primary & 90 & 74.3 & 31 & 25.7 & 121 & 30.25 \\
\hline & Secondary & 92 & 62.1 & 56 & 37.9 & 148 & 37 \\
\hline & Higher secondary & 44 & 80 & 11 & 20 & 55 & 13.75 \\
\hline & Graduate & 20 & 60.6 & 13 & 39.4 & 33 & 8.25 \\
\hline & \multicolumn{5}{|c|}{$\chi^{2}=30.86, \mathrm{DF}=4, \mathrm{p}<0.001$} & 400 & 100 \\
\hline
\end{tabular}

IV. Discussion:

In the present study, Majority $242(60.50 \%)$ women were in the age group 20-24 years followed by 106 (26.50\%) in age group 25-18 years, in the age group of 15-19 years $34(8.50 \%)$ and less in 30-34 years 18 $(4.50 \%)$. A study conducted in Nepal showed that $87.81 \%$ women were less than 30 years. $^{7}$ Other study in Nigeria noted that majority of the women were $30-34$ years $(34.1 \%)$ followed by of $25-18$ years $(33.5 \%), 20-24$ years $(14.8 \%), 35-39$ years $(12.9 \%)$ and rest were in other age group which was different from our study. ${ }^{8}$ One of the study of Kalyobia showed that $30 \%$ women were $18-25$ years, $50 \%$ were $25-32$ years and $20 \%$ were more than 32 years of age. ${ }^{9}$ Majority $148(37.0 \%)$ of the women had secondary level education followed by primary level education $121(30.3 \%)$, higher secondary $55(13.8 \%)$, illiterate $43(10.8 \%)$ and $33(8.3 \%)$ had graduate level education in this study. A study in Orissa revealed that $16.91 \%$ were illiterate, 40.83 were having primary education, $24.16 \%$ were having secondary education and $7.08 \%$ were having higher secondary education and 
above education. ${ }^{10}$ A study in Nepal revealed that $16.7 \%$ were illiterate, $25.8 \%$ were having primary education, $56.1 \%$ were having secondary education and above education. ${ }^{7}$

Maximum number of 187 (74.30\%) were Hindu, 102(25.30\%) were Muslim and 1(0.40\%) were Buddhist in this study. A study in Karnataka showed $67.61 \%$ were Hindu and $25.71 \%$ were Muslim, and 6.66 were others religion followers. ${ }^{11}$ In this study $161(67.70 \%)$ were living in joint family. $117(18.30 \%)$ were living in nuclear family and $12(3.0 \%)$ were living in extended family. A study conducted in Orrisa showed that $77.08 \%$ were living in joint family and $22.92 \%$ were living in nuclear family. ${ }^{10} 55.23 \%$ were living in joint family and $44.76 \%$ were living in nuclear family in the study done in Karnataka. ${ }^{11}$

In this study $346(86.50 \%)$ were housewife, $16(4.0 \%)$ were labor. 38(9.50\%) of respondent used to do other type of work. A study conducted in Kalyobia showed that $58 \%$ were housewife and $42 \%$ were doing other work outside. $^{9} 95.23 \%$ were housewife, $2.85 \%$ were laborers, $1.9 \%$ were Professionals in a study done in Karnataka. ${ }^{11}$ In this study majority of the respondents $162(68.0 \%)$ were non-vegetarian whereas $128(32.0 \%)$ were vegetarian. The respondent $73.33 \%$ were non-vegetarian and $26.66 \%$ were vegetarian in the study done in Karnataka. $^{11}$

Majority 251(62.70\%) had registered their pregnancy in first trimester, 125(31.30\%) had registered their pregnancy in second trimester and $24(6.0 \%)$ had registered their pregnancy in third trimester in this study. In the study done in Nigeria $14.24 \%$ had registered their pregnancy in first trimester, $54.75 \%$ had registered their pregnancy in second trimester and $31 \%$ had registered their pregnancy in third trimester. ${ }^{8}$ In the study done in Karnataka, 56.19\% had registered their pregnancy in first trimester, 36.19\% had registered their pregnancy in second trimester and $9.52 \%$ had registered their pregnancy in third trimester. ${ }^{11}$

Most of the respondents 139 (34.80\%) had family's income Rs.5000-10000 per month. $122(30.50 \%)$ had family's income less then Rs 5000 per month. 83(20.80\%), 37(9.20\%), 15(3.80\%) had family income per month Rs.10000-15000, Rs 15000-20000, Rs20000-25000 respectively. Only 4(1.0\%) had family's income more than Rs 25000 per month in this study. In the study done in Karnataka, $99.05 \%$ had income of less than Rs. 5000 and $0.95 \%$ had income of more than Rs.5000. ${ }^{11}$

In our study, most of women 194(48.50\%) were in first gravid, 133 (33.20\%) were in second gravid. 64 $(16.0 \%)$ and $9(2.30 \%)$ were in third and fourth gravid respectively. A study conducted Iranian $44.44 \%$ were in first gravid, $25.9 \%$ were in second gravid. $14.2 \%$ were in third and $15.5 \%$ in fourth and above gravid. ${ }^{12}$ In Kalyobia study, $18 \%$ were in first gravid, $71 \%$ were in multi gravid. ${ }^{9}$ Out of 400 respondents, $186(46.5 \%)$ didn't have any child. $157(39.30 \%)$ respondents had one child. Similarly 18(7.20\%), 16(6.70\%), 1(0.30\%) had two, three and four children respectively. In the study done in Nigeria, $43.91 \%$ didn't have any child, $28.37 \%$ had one child, $15.24 \%$ had two children, $6.05 \%$ had three children and $6.44 \%$ had four and more than four children. ${ }^{27}$ In Kalyobia study, $50.5 \%$ didn't have any child, $49.5 \%$ one and more than one children. ${ }^{9}$

None of the women who were illiterate gave any correct answer regarding cause of anemia. Majority of correct answers regarding cause of anemia was given by women who were educated up to graduate. The study found that there was significant association between cause of anemia and women's education at $\mathrm{p}<0.001$.

None of the women who were illiterate gave any correct answer regarding sign and symptoms of anemia. Majority of correct answers regarding sign and symptoms of anemia was given by women who were educated up to graduate. The study found that there was significant association between sign and symptoms of anemia and women's education at $\mathrm{p}<0.001$. Most of women had knowledge about proper diet to prevent anemia but majority of correct answer was given by women who was educated up to higher secondary and graduate. The study found that there was significant association between prevent anemia and women's education at $\mathrm{p}<0.001$.

Most of women had knowledge regarding prevention and treatment of anemia but majority of correct answer was given by women who were educated up to higher secondary level. The study found that there was significant association regarding prevention and treatment of anemia and women's education at $p<0.001$. Most of the women were doing healthy practice to prevent anemia but majority of women who were educated up to higher secondary were doing healthy preventive practice to prevent anemia. The study found that there was significant association regarding preventive practice regarding anemia and women's education at $\mathrm{p}<0.001$.

\section{Conclusion:}

The study result showed that knowledge regarding cause of anemia, sign and symptoms of anemia, proper diet to prevent anemia was poor. Knowledge regarding prevention and treatment of anemia, knowledge regarding preventive practice of anemia was good. The result clearly showed that there was significant association between women's education and knowledge regarding cause of anemia, sign \& symptoms of anemia, proper diet to prevent anemia, prevention and treatment of anemia, preventive practice regarding anemia. 


\section{Acknowledgement:}

We are thankful to Dr.(Mrs.)Vijaya Naik, Professor and Head, Department of Public Health, Mr. M.D. Mallapur, Statistician, Department of Community Medicine, Jawaharlal Nehru Medical College, KLE University Belgaum and Dr. Sridevi Metgud, Department of gynaecology and Obstetrics. We thank to all who supported directly and indirectly

\section{Conflict of Interest: NIL}

Funding: No source of funding

\section{References:}

[1]. World Health Organization (WHO). The prevalence of Anemia in women: a tabulation of available information. Geneva, Switzerland: WHO; 1992.( WHO\MCH\MSM\92.2). Assessed on 20/10/2012.

[2]. Wright.D. Anemia in pregnancy. http://www.articlesbase.com/womens-health-articles/anemia-in-pregnancy-1541442.html.dec 4, 2009; Assessed on 2/11/2012.

[3]. Okeke PU. Anemia in Pregnancy-is it a Persisting Public Health Problem in Porto Novo-Cape Verde?. Research Journal of Medical sciences 2011; 5(4):193-99.

[4]. Basvanthappa BT. Community Health Nursing. $2^{\text {nd }}$ ed. New Delhi: Jaypee; 2008.

[5]. Vijaynath K, Patil R, Jitendra, Patel A. Prevalence of anemia in pregnancy. Indian journal of applied basic medical science 2010 july; $12 \mathrm{~B}(15): 45-50$.

[6]. Deoki N. Strategies for effective implementation of National Programmes for prevention and control of anemia in Mothers and Children 2009

[7]. Ghimire N, Pandey N. Knowledge and Practice of Mothers regarding the Prevention of Anemia during Pregnancy, in teaching hospital, Kathmandu. Journal of Chitwan Medical College 2013; 3(5):14-17

[8]. Buseri FI, Uko EK, Jeremiah ZA, Usanga EA. Prevalence and Risk Factors of Anemia Among Pregnant women in Nigeria. The Open Hematology Journal 2008; 2:14-19.

[9]. ElHameed HSA, Mohammed AI, Hameed LTAE. Effect of Nutritional Educational Guideline among Pregnant Women with Iron Deficiency Anemia at Rural Areas in Kalyobia Governorate. Life Sci J 2012; 9(2):1212-17.

[10]. Panigragi A, Sahoo BP. Nutritional Anemia and its Epidemiological Correlates among Women of Reproductive Age in Urban Slum of Bhubaneswar, Orissa. Indian journal of Public health, 2011;55( 4).

[11]. Anitha, M. A study to assess the knowledge and practices regarding prevention of anemia among registered pregnant mothers attending antenatal clinics in selected hospital of Belgaum, Rajiv Gandhi University of Health Sciences, Karnataka, Bangalore 2005

[12]. Moradi F, Mohammadi S, Kadivar AA, Masoumi SJ. Knowledge and practice of pregnant women in fars province about intake of iron supplements. Acta Medica Iranica 2007; 45(4): 301-04 